\title{
La alternativa al conflicto: Punto de Encuentro Familiar
}

\section{The alternative to the conflict: Familiar Meeting Point}

Elena MORTE BARRACHINA ${ }^{1}$ Marisol LILA MURILLO ${ }^{2}$

\section{RESUMEN}

Un Punto de Encuentro Familiar (PEF) es un espacio neutral e idóneo que favorece y hace posible el mantenimiento de las relaciones entre el menor y sus familias cuando, en un proceso o una situación de separación y/o divorcio, o acogimiento familiar, el ejercicio del derecho de visitas se ve interrumpido o bien es de cumplimiento dificil o conflictivo. Es una alternativa positiva a los tradicionales sistemas de intercambio de los hijos establecidos hasta la fecha, puesto que sirve de espacio mediador en la relación entre los progenitores y favorece la interacción paterno-filial, preservando los derechos del menor y el derecho de los padres a ejercer como tales. En el presente artículo se intenta dar a conocer qué es un PEF, cómo surge esta iniciativa, y cuál es su demanda social en la actualidad.

\section{PALABRAS CLAVE}

Punto de Encuentro, Recurso social, Derecho de visitas, Familia, Mantenimiento de las relaciones, Progenitor no custodio, Menor.

\section{ABSTRACT}

This paper describes a Family Meeting Point. It is conceived as a neutral and appropriate space that favours and makes possible the maintenance of the relationships between the

\footnotetext{
${ }^{1}$ Licenciada en Psicologia (CV8535). Estudiante de Tercer Ciclo de Psicología Social. Universidad de Valencia. emorba@alumni.uv.es

${ }^{2}$ Profesora titular de Psicologia Social de la Familia. Universidad de Valencia. marisol.lila@uv.es
} 
minor and his family when, in a situation of separation, divorce, or foster families, the exercise of the right of visits is interrupted, or its fulfillment is difficult or conflictive. It is a positive alternative to the traditional systems of interchange established to date. In view of the fact that the possible conflict and tension in the relations between progenitors, it serves as mediating space in the relations and supports the interaction between non custodial parent and the minor, preserving his rights and well-being. The present article tries to explain what a meeting point is, how this initiative appeared, and which its social demand is at the present time.

\section{KEY WORDS}

Meeting point, Social resource, Right of visits, Family, Maintenance of the relationships, Non-custodial parent, Minor. 
Desde que, hace 10 años, se puso en funcionamiento el primer Punto de Encuentro Familiar (PEF) en España, este servicio ha ido adquiriendo mayor protagonismo en nuestro contexto sociocultural. La demanda y necesidad de este servicio ha ido en aumento, convirtiéndose en una alternativa a la vía judicial en la reducción del conflicto familiar (Sacristán, 1999).

No obstante, en otros países de la Unión Europea, los PEF se encuentran en funcionamiento desde la década de los 80. Francia es uno de los países que cuenta con un mayor desarrollo de estos servicios, puesto que ya cuenta con puntos de encuentro para el mantenimiento de las relaciones entre padres e hijos en todos sus distritos ${ }^{1}$. Los primeros Puntos de Encuentro o Point-Rencontre fueron creados a finales de los años 80 , gracias a iniciativas locales en Burdeos, Grenoble y Clermont-Ferrand. Además, en los $\mathrm{PEF}$ franceses se enfatiza la concepción de este como instrumento para reestablecer y mantener las relaciones paterno-filiales, en contraposición a los PEF estadounidenses, centrados en la supervisión de las visitas, así como en que tengan lugar en un espacio seguro (Bastard, 2001).

En este sentido se puede distinguir entre aquellos que centran su intervención entorno a la seguridad de los niños (PEF de Estados Unidos, Canadá, Australia y Nueva Zelanda) y aquellos que centran su interés en favorecer la relación entre padre no custodio e hijo (PEF de Francia, Suiza, Bélgica y Québec) (Sacristán, 1999).

El principal objetivo de este artículo es dar a conocer qué es un Punto de
Encuentro Familiar y a qué tipo de demanda social responde. Para ello, se desarrolla la contextualización de este recurso dentro de nuestro marco social, la aparición e incidencia de los PEF en España, y los documentos que regulan estos servicios. Además, ofrece una definición de los PEF y se tratan algunos aspectos formales, tales como los objetivos del servicio, tipos de usuarios, intervenciones y metodología de trabajo.

\section{APARICIÓN E INCIDENCIA DE LOS PEF EN ESPAÑA}

Desde el año 1981, en que se promulgó la ley que modificaba la regulación del matrimonio y determinaba el procedimiento a seguir en los casos de nulidad, separación y divorcio, se ha ido registrando un aumento gradual y sostenido de separaciones. Según los datos del Consejo General del Poder Judicial, en 2003 se resolvieron 53.700 separaciones de mutuo acuerdo y 25.723 contenciosas, junto a 28.172 divorcios consensuados y 19.147 no consensuados ${ }^{2}$. En España, el 90\% de las mujeres y hombres que se separan tienen hijos. Más de la mitad tiene uno o dos hijos. Respecto a la edad de los hijos, uno de cada tres hijos de familia separada tiene menos de cinco años, y uno de cada cinco ronda la mayoría de edad o la sobrepasa (Romero, 2002). Una de las consecuencias de este aumento de las separaciones ha sido el incremento de la demanda de recursos y apoyo a la familia, entre los que destacan los servicios de mediación familiar y los puntos de encuentro (Artamendi, 2004). La primera iniciativa surgió en 1994 con la Asociación para la Protección del Menor en los Procesos de Separación de sus Progenitores (APROME),

\footnotetext{
${ }^{1}$ Para más información ver: www.mediation-familiale.info/carte_mediation.html.

${ }^{2}$ Fuente: Instituto Nacional de Estadística (INE)
} 
con el objeto de que la patria potestad se ejerciera siempre en beneficio de los hijos y ofreciendo una alternativa de intervención en los conflictos familiares. APROME desarrolló en 1994 el primer Punto de Encuentro de España en Valladolid. Su principal objetivo era salvaguardar la integridad del menor.

Con anterioridad a la aparición de los $\mathrm{PEF}$, muchas familias se encontraban en contextos fríos, como Comisarías de Policía u otro tipo de Oficinas. El PEF es el primer contexto normalizado con que cuentan las personas para ensayar alternativas no contenciosas para resolver sus diferencias (Goikoetxea, Gutiérrez, Lizarraga et al., 2003).

Debido a la creciente demanda y necesidad de este servicio, se han ido instaurando progresivamente nuevos $\mathrm{PEF}$ por toda la geografia española. Las Administraciones Públicas y el Consejo General del Poder Judicial han reconocido su utilidad y eficacia en la resolución de conflictos familiares y se pretende que exista un Punto de Encuentro Familiar en cada capital de provincia ${ }^{3}$. En la Jornada sobre Puntos de Encuentro Familiar, celebrada en la Sede del Consejo General del Poder Judicial el 28 de septiembre de 2000 , donde participaron representantes de la administración, jueces, fiscales, psicólogos y responsables de los PEF, se redactaron una serie de conclusiones entre las que destacan: (1) necesidad de que el recurso se extienda a todas las ciudades de España, adaptando el número de servicios y la duración de los mismos al censo de su población; (2) necesidad de dotar estos centros con una financiación suficiente; (3) necesidad de prevención y reparación, por parte de las Administraciones Públicas, de los conflictos entre los adultos derivados de los procesos de ruptura familiar y que constituyen situaciones de riesgo para los menores, por medio del establecimiento de servicios, como los Puntos de Encuentro Familiar; (4) necesidad de un reglamento de Régimen Interior que regule su funcionamiento con el máximo detalle; (5) importancia de los PEF, como recurso social, en la intervención en situaciones de violencia doméstica y maltrato, y (6) deseabilidad de una legislación autonómica o estatal sobre los $\mathrm{PEF}^{4}$.

En consonancia con estas conclusiones, cabe destacar la mención que se realizó sobre los PEF en el seminario Encuentro de jueces y abogados de familia: Incidencia de la ley de enjuiciamiento civil en los procesos de familia, que tuvo lugar en noviembre de 2003 en Madrid. A modo de resumen, en este encuentro se destaca la necesidad de su expansión y la promoción de una mayor operatividad y funcionamiento. Del mismo modo, se propone que las Administraciones Públicas se impliquen en la regulación de estas entidades, en la homologación o formación para la creación del Punto de Encuentro y en que exista un control y colaboración entre el PEF y el Juzgado de Familia.

En las Jornadas sobre Mediación Familiar y Puntos de Encuentro Familiar: perspectivas jurídicas y sociales ${ }^{5}$, cele-

\footnotetext{
${ }^{3}$ Ver Conclusiones de la jornada sobre puntos de encuentro familiar celebrada en la sede del Consejo General del Poder Judicial el 28 de septiembre de 2000, en http://www.derecho-familia.com/secciones/legislacion/legislacion/ViolenciaMujeres2.pdf

${ }^{4}$ No obstante, se estima que los artículos 94 del Código Civil, los artículos 923 y 924 de la Ley de Enjuiciamiento Civil y el artículo 17 de la Ley Orgánica del Poder Judicial, dan cobertura legal a la colaboración de los PEF con los Juzgados de Familia.

${ }^{5}$ Disponible en la base de datos digital Sepinnet (Referencia: SP/DOCT/1897).
} 
bradas en enero de 2004 en Valladolid, se alcanzaron las siguientes conclusiones sobre los PEF: (1) necesidad de dotarlos de un marco jurídico, tanto de una regulación externa, como de una regulación interna a través de Reglamentos de régimen interno sobre aspectos relacionados con la financiación, los requisitos, la especialización del personal, las facultades y necesidades mínimas de sus locales; (2) mejorar la coordinación entre los PEF y los Juzgados, considerando la necesidad de crear un Protocolo unificado de derivación de supuestos familiares a los PEF desde los órganos judiciales o administrativos; (3) manifestar las dificultades existentes para obligar a cumplir las resoluciones judiciales a aquellos progenitores que se niegan a acudir a los PEF, insistiendo en la necesidad de contar con una normativa específica para la ejecución de las resoluciones judiciales en materia de familia; y, (4) resaltar supuestos como la conveniencia de que los Juzgados no remitan casos cronificados, la participación positiva en las intervenciones de la familia extensa y la necesidad de derivar a grupos familiares a que reciban apoyo terapéutico. Asimismo, en estas Jornadas se destaca el aumento de casos atendidos en los PEF, tras la entrada en vigor el mes de agosto de 2003, de la Orden de Protección de las Víctimas de la Violencia Doméstica. Resaltan la idoneidad de este recurso en los supuestos de violencia doméstica y de género.

\section{DOCUMENTOS REGULADORES DE LOS PUNTOS DE ENCUENTRO FAMILIAR}

Existe un documento marco creado por especialistas que tiene validez para todos los PEF. Se trata de la Carta Europea de los Puntos de Encuentro para el mantenimiento de las relaciones entre los hijos y sus padres (Carta Europea,
2004). La redacción de la Carta Europea de Puntos de Encuentro se inició en la reunión que tuvo lugar en París, en el año 2001. Pretendía establecer acuerdos acerca de temas como la formación, el estatus legal, la relación con la justicia y otros profesionales, el papel de la Mediación Familiar, criterios de selección de casos y otros aspectos. Hasta la Carta Europea de 2004 no se producen cambios notables. En esta carta se especifica el objetivo y finalidad de estos lugares. Considera que la acción de los Puntos de Encuentro se basa en el reconocimiento del vínculo de filiación y en el interés y el derecho del menor en ver aseguradas la instauración y la continuidad de todas las relaciones necesarias para la construcción de su identidad. Esta se sitúa en la articulación de las dimensiones psicológica, social y jurídica. En cualquier situación, esta acción encuentra su límite en el interés superior del menor $\mathrm{y}$ en el respeto de su seguridad física, psíquica y moral. Además, se especifica que la acción de los Puntos de Encuentro se inscribe en el respeto de los textos legislativos en vigor de cada país, de la "Convención sobre las relaciones personales referidas a los menores" del Consejo de Europa y de la "Convención Internacional de los Derechos del Niño". Otro de los cambios a destacar en la redacción de la última Carta Europea de 2004, es la sustitución del término "familiar", por "para el mantenimiento de las relaciones hijos-padres", abriéndose un debate respecto a la nomenclatura de este servicio, cuyo cambio ya se había propuesto en la Carta Europea del año 2003. En Francia, por ejemplo, la utilización del término Punto de Encuentro (Point-Rencontre) es poco habitual, y en ningún momento se utiliza el término "Familiar". Optan por denominar a estos servicios como Lugar de Encuentro para ejercer el derecho de visitas (Lieu d'accueil pour l'exercise du droit de visite). Este debate podría considerarse una 
consecuencia de los problemas que plantea la definición del concepto de "familia"(Alberdi, 1999). Ahora mismo, el modelo estereotipado de familia tradicional está siendo sometido a un proceso de redefinición y la diversidad de la vida familiar no puede reducirse a una única definición. Más bien éstas dependen del marco teórico y del contexto sociocultural del momento (Gracia y Musitu, 2000).

Centrándonos en la existencia de algún tipo de decreto o proyecto de ley que sistematice los objetivos a cubrir por los PEF, es necesario destacar que en algunas comunidades autónomas, tales como Asturias y Navarra, ya han redactado un marco normativo básico. Asturias ya dispone de un borrador de Decreto por el que se regula el régimen juridico de los Puntos de Encuentro Familiar. En este se especifican las disposiciones generales de este servicio, el ámbito de actuación de los PEF, el procedimiento de intervención del punto de encuentro, su funcionamiento (normas y seguridad), cómo ha de ser la organización (estructuras, coordinación y equipo técnico) y cómo ha de ser el protocolo de derivación por la autoridad al Punto de Encuentro Familiar ${ }^{6}$. Por otro lado, Navarra optó por clasificar el servicio de Punto de Encuentro Familiar, junto con el de Centro de Día Infantil y Juvenil, en la Orden Foral 18/2002, de 20 de febrero, dotando al PEF de una marco normativo. La necesidad de contemplarlos como nuevos servicios debido a que no podian incluirse en ninguno de los preexistentes, obligó a proceder a la clasificación individualizada de los mismos en el Decreto Foral 209/1991, de 23 de mayo. La puntualización que se realiza al respecto del Punto de Encuentro se desarrolla en el
Anexo del Decreto, y contempla únicamente aspectos concretos como la definición del PEF, tipo de personas usuarias, servicios prestados, personal y condiciones específicas sobre el centro (Boletín Oficial de Navarra, nº41, 2002).

\section{Definición y objetivos de los Puntos de Encuentro Familiar}

El Punto de Encuentro Familiar se desarrolla como una alternativa de intervención temporal, realizada en un lugar idóneo y neutral, donde se produce el encuentro de los miembros de la familia en crisis, atendidos por profesionales debidamente formados, facilitando la relación paterno-filial y garantizando la seguridad y el bienestar del menor y del padre / madre vulnerable. Según la Asociación para la protección del menor en los procesos de separación de sus progenitores $(\mathrm{APROME})^{7}$, dos son los objetivos generales: (1) favorecer el cumplimiento del derecho fundamental del menor de mantener la relación con ambos progenitores después de la separación, estableciendo los vínculos necesarios para su buen desarrollo psíquico, afectivo y emocional y, (2) preparar a los padres para que consigan autonomía y puedan mantener las relaciones con sus hijos sin depender de este servicio.

Del mismo modo, APROME establece los siguientes objetivos específicos: (1) garantizar que el cumplimiento del régimen de visitas no suponga una amenaza para la seguridad del niño o del padre / madre vulnerable; (2) facilitar el encuentro del hijo con el progenitor que no tiene la custodia y con la familia extensa de este; (3) permitir a los menores expresar

\footnotetext{
${ }^{6}$ Véase Borrador de decreto por el que se regula el régimen jurídico de los PEF en el Principado de Asturias, 2003.

${ }^{7}$ Véase http://www.aprome.org
} 
sus sentimientos y necesidades, sin temor a que sean contrarios a lo indicado por sus padres; (4) evitar el sentimiento de abandono del menor; (5) facilitar orientación profesional para mejorar las relaciones paterno / filiales y las habilidades de crianza parentales, y (6) disponer de información fidedigna sobre las actitudes y aptitudes parentales que ayude a defender en otras instancias administrativas o judiciales -si fuera necesario-, los derechos del niño.

En resumen, este recurso social está orientado principalmente a padres separados y/o divorciados y parejas separadas sin ningún tipo de vínculo formal legalizado que, debido a la existencia de conflictos, encuentran dificultades para ejercer con éxito la coparentalidad. También puede recurrirse a los Puntos de Encuentro para proceder al mantenimiento de la relación del menor con la familia extensa y, en casos en que se establece la necesidad de que el menor se revincule con su familia biológica, cuando está conviviendo con una familia en régimen de acogida. En definitiva, es una alternativa positiva a los tradicionales sistemas de intercambio de los hijos establecidos hasta la fecha, puesto que debido al posible conflicto y tensión en las relaciones entre los progenitores, sirve de espacio mediador en la relación y favorece la interacción paterno-filial, preservando los derechos del menor y de los padres a ejercer como tales (Sacristán, 1999).

\section{Los usuarios de los Puntos de Encuentro Familiar}

A los Puntos de Encuentro se accede por decisión judicial, por derivación de los servicios sociales y por derivación de otros servicios especializados, principalmente de protección del menor y de la mujer. Generalmente, el cumplimiento del régimen de visitas a través del Punto de Encuentro Familiar se produce por decisión de: (1) el Juez de Familia o de su equipo técnico cuando se detectan situaciones de riesgo, o a instancia del progenitor que no tiene la custodia y le resulta imposible que se cumpla el régimen de visitas. En este caso será mediante providencia dictada por el Juez de Familia acordando que el equipo técnico informe sobre dicha solicitud; (2) el Juez de Instrucción, que en aplicación de la Orden de Protección de las víctimas de la violencia doméstica adopta medidas civiles $\mathrm{y}$, entre ellas, el régimen de visitas. En menor medida, también acceden usuarios por acuerdo entre ambos progenitores y el Punto de Encuentro, aunque estos casos son los menos frecuentes.

Según APROME, los usuarios que asisten al centro por derivación explícita se encuentran en alguna o varias de las situaciones siguientes: (1) niños separados de sus padres con medidas de protección que residen con una familia acogedora, o con la familia extensa y que, por decisión judicial, se encuentran en proceso de revinculación con su familia biológica; (2) hijos de madres que residen en casas de acogida o protección, porque han sufrido algún tipo de maltrato y es necesario mantener la confidencialidad del domicilio; (3) progenitores que por circunstancias personales (están en tratamiento de enfermedades o toxicomanías, no conocen al hijo o carecen de habilidades para ejercer el cuidado del menor) no pueden ejercer el derecho de visitas sin que sean supervisadas, ya que puede representar algún riesgo para la integridad del menor; (4) progenitor no custodio que carece de un lugar que garantice el bienestar del menor durante la visita porque reside fuera de la ciudad, su vivienda no reúne condiciones adecuadas o convive con personas que ejercen influencias negativas o suponen 
riesgo para el menor; (5) familias en las que el progenitor custodio se opone a la entrega del menor y no favorece los encuentros, así como aquellos menores que muestran una disposición negativa a relacionarse con su progenitor, y (6) familias que, durante y después del proceso de separación, tienen conflicto o dificultad para mantener la relación entre los hijos menores y ambos progenitores.

En todos los casos, es necesario que se aporte una documentación específica para poder realizar un seguimiento del caso y comprobar el estado actual de la pareja, o en su caso del progenitor custodio y del adulto que procede a realizar las visitas, ya sea familia extensa 0 padres biológicos. Generalmente, los usuarios derivados de Servicios Sociales aportan una ficha bastante completa, donde se incluye información sobre el núcleo de convivencia actual del menor, la familia biológica del menor autorizada a visitas y datos de identificación, los datos de identificación completos del menor, antecedentes y observaciones de la historia familiar, la tipologia de las visitas y cual es el objetivo de las mismas. Conjuntamente aportan todo tipo de información que pueda ser útil para alcanzar los objetivos planteados, como son sentencias judiciales de separación, medidas provisionales y órdenes judiciales de cualquier tipo. Además, en cada expediente que se abre en el Punto de Encuentro se adjuntan otros documentos, para llevar a cabo un seguimiento estricto de las visitas: (1) fotocopias del DNI de la persona que ostenta la custodia, ya sea temporalmente por régimen de acogida o por asignación judicial, y de la persona que ejerce el derecho de visitas; (2) registro de entrevistas, para la recopilación de información biográfica e historia familiar; (3) registro de asistencias y faltas; (4) informes aportados por los Servicios Sociales u otros, como sen- tencias; (5) normativa del Punto de Encuentro firmada por cada parte; y (6) ficha del control de las visitas, ya sean tuteladas o sean intercambios.

En principio, a partir de la experiencia de determinados Puntos de Encuentro, en los casos procedentes de Servicios Sociales se establecen inicialmente visitas mensuales de entre 2 horas y 1 hora y que posteriormente se van ampliando, según la evolución del caso. A los casos que proceden de Juzgados se les asignan visitas quincenales, que van ampliando gradualmente según se desarrolle satisfactoriamente la relación paterno-filial. Los casos derivados al PEF, desde los Servicios de Protección a la Mujer y al Menor, suelen reducirse a intercambios de fines de semana y periodos vacacionales.

\section{Tipos de visitas en los Puntos de Encuentro Familiar}

En el PEF se pueden realizar dos tipos de visitas, además de la función de recogida y entrega (o intercambio). La convivencia en el centro se realiza principalmente en los casos de reiniciación, tras un periodo de suspensión, de las relaciones paterno o materno filiales, cuando éstas nunca se hubieran desarrollado con normalidad, así como en aquellas situaciones en que el progenitor no custodio presenta factores de riesgo que requieran supervisión (Sepúlveda y Serrano, 2000).

Los servicios de visita tutelada requieren la presencia de un profesional en todo momento. Dicho profesional se encarga de vigilar y apoyar a padres 0 visitantes en el ejercicio de sus roles y tareas. Su actuación queda siempre supeditada al interés del menor. Los servicios de visita no tutelada transcurren en el interior del centro, sin la presencia 
de un profesional. Muchas veces, si cuentan con permisos judiciales, pueden desarrollar la visita en el exterior del centro. El objetivo principal del servicio de recogida y entrega es garantizar que el régimen de visitas se realice. Por ello, los profesionales reducen su labor a constatar que los intercambios del menor se realizan en las condiciones estipuladas por la sentencia judicial pertinente. Los visitantes pueden salir del centro en compañia del menor.

Siempre se sigue el mismo procedimiento cuando se realizan las visitas y los intercambios. El progenitor custodio, o tutor legal, deja al menor en el centro, y el otro progenitor, o persona que ejerce el derecho de visitas, lo recoge (gestión de impasse). Esto siempre ha de realizarse evitando el contacto físico y visual entre ambos progenitores, por lo que se establecen unos horarios para evitar que ambos coincidan en las dependencias del punto de encuentro. En el PEF de Murcia $^{8}$ se procede del siguiente modo: se cita al progenitor no custodio diez minutos antes de la hora en que deba llevarse a cabo la visita y, a la hora establecida, llega el progenitor custodio y el menor/es. Acto seguido el progenitor custodio abandona el PEF, permaneciendo en el Servicio el no custodio en compañia del menor/es, quienes transcurridos diez minutos podrán abandonar el Servicio. Para la entrega del menor al progenitor custodio se seguirán las mismas pautas, llegando primero el no custodio con el menor, permaneciendo en el PEF hasta que llega el custodio, recoja al menor y abandone el mismo, y transcurridos diez minutos desde que el custodio y el menor abandona el Servicio podrá abandonarlo él.
Otro aspecto que hay que cuidar especialmente es el contexto físico donde se producen estas visitas. Las dependencias del Punto de Encuentro han de cubrir todas las necesidades que puedan surgir dentro de una convivencia entre el progenitor no custodio, o familiar que ejerce las visitas, y el menor. Este hecho es importante porque en un régimen de visitas normalizado deben incluirse rutinas cotidianas de convivencia, tales como dormir, comer, aseo y vinculación con la familia extensa, así como realizar celebraciones de cumpleaños y otras festividades. Esto hace imprescindible la existencia de determinadas dependencias y espacios libres en estos centros, destinados al juego y a actividades concretas. Asimismo, ha de reunir toda una serie de recursos materiales que faciliten el mantenimiento de las relaciones. Además, esto ha de combinarse con un ambiente cálido y acogedor que no entorpezca el desarrollo y bienestar del menor.

\section{Metodologia de trabajo en los Puntos de Encuentro Familiar}

Actualmente no existe ningún protocolo de actividades e intervención común para los Puntos de Encuentro. La Asociación para la Protección del Menor ${ }^{9}$ (APRO$\mathrm{ME}$ ) propone cinco tipos de actividades a llevar a cabo dentro del centro en diferentes momentos, desde la entrada hasta la salida del PEF: (1) valoración del sistema familiar en relación al cumplimiento del derecho de visitas; (2) apoyo al cumplimiento del régimen de visitas; (3) intervención Psicosocial Individual y familiar; (4) mediación para facilitar la independencia del servicio; y, (5) elaboración de registros y documentación.

\footnotetext{
${ }^{8}$ Véase http://www.carm.es/ctra/politicasocial/menoryfamilia/programas/pef.asp

${ }^{9}$ Véase http://www.aprome.org
} 
La metodología de actuación varía según la problemática del caso, y cada intervención obedece a un plan de acción personalizado, con un enfoque integral biopsicosocial. Para continuar, interrumpir o finalizar la permanencia en el centro se atenderá a los dictámenes emitidos por el equipo técnico, el cual presentará informes con cierta periodicidad a la entidad derivante, para dar a conocer la evolución del caso (Sepúlveda y Serrano, 2000).

La metodología de trabajo que siguen la mayoría de los PEF se resume en la Tabla 1:

En cada una de estas fases se emplean unas herramientas de trabajo en función de las necesidades que se plantean. Asimismo, a lo largo de todo el proceso se facilita asesoramiento jurídico-social a las familias y apoyo psicopedagógico al menor.

En la Fase de Contacto, se hace un estudio del caso derivado al punto de encuentro. Se valora la problemática del caso, así como la motivación del progenitor no custodio para relacionarse con los hijos, del progenitor custodio para que el hijo se relacione con el otro y del menor, si es posible. Se recopilan todos los datos de identificación posibles y se procede a realizar una valoración del sistema familiar con los informes disponibles. Con todos estos datos se abre un expediente individualizado, donde se llevará el registro de todo lo que acontezca durante la evolución del régimen de visitas. El último paso consiste en la primera toma de contacto telefónico con los progenitores,

Tabla 1. Metodologia de trabajo en el PEF/ Work methodology in familiar meeting point

\begin{tabular}{|c|c|}
\hline 1. Fase de Contacto & $\begin{array}{ll}\text { - } & \text { Estudio del Protocolo de Derivación. } \\
\text { - } & \text { Apertura de expediente. } \\
\text { - } & \text { Primera toma de contacto telefónico para concertar cita. }\end{array}$ \\
\hline 2. Fasc Inicial & $\begin{array}{ll}\text { - } & \text { Familiarización con las instalaciones y adaptación. } \\
\text { - } & \text { Primcra cntrcvista (anamncsis). } \\
\text { - } & \text { Información sobrc rcglamcnto intcrno y aclaración de } \\
\text { inccrtidumbres. } \\
\text { - } \quad \text { Estudio dcl caso y cvaluación. } \\
\text { - Elaboración del Programa de Intervención por el equipo } \\
\text { técnico. } \\
\text { - Intervención previa al inicio del régimen de visitas, si se } \\
\text { considera oportuno. }\end{array}$ \\
\hline $\begin{array}{l}\text { 3. Fasc de } \\
\text { Intcrvención }\end{array}$ & $\begin{array}{ll}\text { - } & \text { Inicio y cumplimicnto del régimen de visitas. } \\
\text { - } & \text { Gestión de recepción de las visitas. } \\
\text { - } & \text { Obscrvación de las visitas y de la intcracción. } \\
\text { - } & \text { Análisis y scguimicnto del caso. } \\
\text { - } & \text { Intervención psicosocial en determinados casos. } \\
\text { - } & \text { Apoyo psicopedagógico. } \\
\text { - } & \text { Registros del desarrollo de la visita y parte de incidencias. }\end{array}$ \\
\hline 4. Fase Final & $\begin{array}{ll}- & \text { Normalización del vínculo paterno-fïlial. } \\
\text { - } & \text { Mcdiación para la indcpendencia del scrvicio. }\end{array}$ \\
\hline
\end{tabular}


con objeto de concertar la cita para realizar la entrevista de valoración.

En la Fase Inicial, antes de iniciar el cumplimiento del régimen de visitas, y para garantizar un clima de seguridad y confianza, se lleva a cabo una primera entrevista con visita al centro, tanto con el menor como con ambos progenitores y familias biológicas 0 acogedoras, en su caso. Las entrevistas se realizan de modo individual y siempre en dias diferentes, para evitar el contacto entre las partes. El menor permanece durante un tiempo jugando o realizando otra actividad, bajo la supervisión de un técnico, para familiarizarse con el ambiente y con los profesionales del PEF. También se le explica por qué está allí y en qué consistirá el encuentro. La finalidad de la entrevista a ambos progenitores es el análisis de la estructura y funcionamiento general de la familia (genograma) antes de la separación y de la nueva situación familiar de cada progenitor. Asimismo, se cuestionan aspectos relacionados con las familias extensas, su implicación en la separación, así como aspectos relacionados con la salud, características psicológicas y aspectos educativos. En la medida de lo posible, se intenta realizar una exploración anamnésica. A lo largo de la entrevista, para la cual se pueden emplear varios días, se explica cuál es la función del PEF, su finalidad, y se resuelven todas las dudas y expectativas que puedan surgir. A partir de este segundo contacto con las partes, se procede a la evaluación del caso, para desarrollar un plan de intervención, el cuál depende de las características valoradas, tales como la voluntariedad, la motivación y la existencia de conflicto latente entre los excónyuges o las partes. En función de estos aspectos, se procede a desarrollar una intervención dirigida al foco del problema observado, si lo hubiese. El plan de intervención que se desarrolla puede ser de dos tipos: (1) intervención indirec- ta en las problemáticas que vayan surgiendo, como consecuencia de la convivencia y cumplimiento del régimen de visitas. Dentro de esta intervención se puede incluir la orientación y asesoramiento familiar, (2) intervención directa, cuando se valora la existencia de algún problema focalizado que requiere de una intervención anterior al inicio del cumplimiento del régimen de visitas. Para este tipo de intervenciones se deja un margen de seis meses para proceder al inicio de las visitas. Generalmente, se produce cuando una de las partes o el menor muestra una actitud oposicionista y negativa a la asistencia al PEF. Durante la permanencia en el PEF, se realizarán todas aquellas intervenciones que se estimen oportunas y siempre atendiendo al beneficio del menor.

En la Fase de Intervención, se inicia el cumplimiento del régimen de visitas, asî como su seguimiento, como ya se ha explicado con anterioridad. Para intentar evitar el conflicto entre los progenitores, se evita su encuentro físico. De cada visita, se lleva un registro sistematizado y se toma nota de: la asistencia y puntualidad, la actitud del niño con el progenitor que ejerce el derecho de visita, la actitud de cada progenitor con el niño, el grado de colaboración del progenitor que ejerce la custodia, los sentimientos del niño y deseos de un nuevo encuentro, comentarios adicionales de los progenitores, otras personas o familiares que han acudido a la visita y aquellas observaciones que se estimen de interés. En función del tipo de visita, se realiza la observación y seguimiento de la misma. Se lleva a cabo la intervención en aquellos casos que se considere oportuno. En todo momento, se presta apoyo al cumplimiento de las visitas. Siempre que es necesario, han de facilitarse habilidades sociales para mejorar la comunicación paterno-filial e intentar fomentar, en la medida de lo posible, la relación afectiva mediante 
juegos o actividades. Otra acción que se puede llevar a cabo radica en apoyar el cumplimiento de las visitas, intentando facilitar el cierre del encuentro y la despedida entre el menor y padre / madre, disminuyendo la angustia de separación, y realizando las intervenciones precisas si se produjesen situaciones de crisis 0 desbordamiento emocional.

En última instancia, es de gran utilidad la mediación para facilitar la independencia del servicio. Esta herramienta se emplearía cuando se ha conseguido la revinculación entre el progenitor no custodio y el menor, y es posible que ambos progenitores lleguen a un acuerdo y respeto en lo que atañe al cumplimiento del régimen de visitas. El trabajo del mediador consistirá en ayudar a que la expareja resuelva sus conflictos y en facilitar la comunicación, en orden a que sean los propios padres los que lleguen a decisiones constructivas, a acuerdos satisfactorios, viables, válidos, duraderos y recíprocamente aceptables. Estos acuerdos deben ser duraderos y tener en cuenta las necesidades de la propia pareja y de los hijos, favoreciendo una relación familiar postdivorcio (Ripol-Millet, 2001).

\section{Conclusiones y Sugerencias}

En las relaciones familiares tras la separación y/o divorcio, uno de los aspectos que más conflicto genera es el régimen de visitas y comunicaciones de los hijos con sus progenitores y la familia extensa. Por ello, el Punto de Encuentro se constituye como una alternativa al conflicto que, ante todo, intenta preservar la integridad física y afectivo-emocional del menor. Este recurso social persigue proteger al menor de un conflicto que no le pertenece y puede repercutir negativamente en su desarrollo madurativo y en la formación de su identidad.
En este trabajo se ha intentado describir qué son los Puntos de Encuentro Familiar y cuál es su finalidad. Con ello, se ha intentado dar a conocer la existencia de un recurso social que permite el mantenimiento de regimenes de visitas conflictivos y que se instaura como el primer espacio con que cuentan las personas para buscar alternativas no contenciosas para resolver sus conflictos. Actualmente, existen determinados aspectos sobre los PEF que suscitan múltiples interrogantes, tales como la concepción del servicio como medida disuasoria del conflicto, la metodología de trabajo que se emplea, el tipo de profesionales que forma el equipo de trabajo, qué formación han de reunir y la necesidad de subvenciones, junto con un marco normativo que regule el servicio.

En primer lugar, es necesario partir de la base de que el PEF es un servicio que se presta como medida disuasoria del conflicto. Ha de concebirse como una disposición cautelar que evita la extensión del conflicto, e intenta facilitar el mantenimiento de la relación entre el padre no custodio y el menor, salvaguardando así el ejercicio de la patria potestad. En este contexto surge el debate acerca de cual es la metodología de trabajo más adecuada, y qué tipo de relación padres-hijos debería promoverse. Los puntos de encuentro franceses, que confían en la mediación como herramienta básica de trabajo, presuponen que la puesta en práctica del derecho de visitas, de forma exitosa, sólo puede obtenerse mediante la reducción del conflicto latente entre los padres. Desde esta perspectiva, se asume que los menores se benefician de la mejora de las interacciones entre los padres separados. Sin embargo, en otros puntos de encuentro del mismo país refutan el uso de la mediación, y consideran que no es factible ni útil el esfuerzo por mejorar la relación entre los padres. Desde este punto 
de vista, únicamente se pretende reestablecer los vínculos entre el padre no custodio y su hijo. Del mismo modo, se considera que el contacto establecido ejercerá cierta influencia en las partes implicadas (Bastard, 2001).

Otro aspecto a considerar está vinculado al tipo de profesionales que ha de trabajar en un Punto de Encuentro y el tipo de formación necesario para poder desempeñar una labor eficaz. En algunos $\mathrm{PEF}$ consideran necesario la existencia de equipos multidisciplinares, como es el caso del de Uribarri (Bizkaia, España) donde colaboran educadores, psicólogos, trabajadores sociales e, incluso, abogados. No obstante, no existe ningún acuerdo en este sentido, y cada PEF cuenta con los profesionales que considera oportuno. La carencia de un protocolo de intervención y funcionamiento de un PEF, así como de la delimitación de la formación necesaria para los técnicos del mismo, podría generar disfunciones en el desempeño de roles. Tal vez, sería interesante promover la creación de una formación específica para los profesionales de un $\mathrm{PEF}$, donde se fomentara el conocimiento interdisciplinario en todas aquellas áreas relativas a la intervención psicosocial, al trato con familias y aspectos jurídico-legales.

Pero ante todo, existe un factor fundamental para promover el desarrollo de este servicio, como es la necesidad de recursos económicos. Dotando de más ayudas a estos servicios, se podrian promover nuevas actividades dentro de los PEF, destinadas a fomentar la coparentalidad, tales como grupos de trabajos para compartir experiencias entre los usuarios y escuelas de padres para promover las habilidades de crianza y parentalidad. Del mismo modo, también se podrian generar iniciativas destinadas a la optimización del funcionamiento del centro, a mejorar la calidad del servicio y a promover la formación especializada del personal técnico. También sería necesaria la creación de un marco jurídico que regulara el servicio a todos los niveles, creando un reglamento interno común y una normativa externa para todos los puntos de encuentro. De esta manera, se podrían generar protocolos de actuación que fomentaran la coordinación entre los órganos derivantes y los Puntos de Encuentro Familiar. 


\section{BIBLIOGRAFÍA}

Alberdi, I. (1999). La nueva familia española. Madrid: Taurus.

Artamendi, R., Estebaranz, L., Hamparzoumian, I. y Pescador, F. M. (2004). Propuestas para una Estrategia de Promoción de los Derechos de la Infancia. Ávila: Conferencia de Infancia y Familias.

Bastard, B. (2001). Contact centres in France. Practices and guidelines-an overview. Supervised Visitation and Child Access: Standards and Intervention Approaches in an international perspective. Munich.

Bolaños, I. (1995). Mediación familiar en procesos contenciosos de separación y divorcio. Mediación: una alternativa extrajurídica. Madrid: Colegio Oficial de Psicólogos.

Carta Europea de los Puntos de Encuentro para el mantenimiento de las relaciones hijospadres (2004). Ginebra.

Conclusiones de la Jornada sobre Puntos de Encuentro Familiar, celebrada en la sede del Consejo General del Poder Judicial el 28 de septiembre de 2000 .

Goikoetxea, J., Gutiérrez, J., Lizarraga, E., Saitua, G. y Novales, A. (2003). Punto de Encuentro de Uribarri. Gizaberri: Asociación Profesional de Educadores Sociales de Euskadi, ㄲo26.

Gracia, E. y Musitu, G. (2000). Psicología Social de la Familia. Barcelona: Paidós.

Musitu, G. y Allatt, P. (1994). Psicosociología de la Familia. Valencia: Albatros Educación.

Ripol-Millet, A. (2001). Familias, trabajo social y mediación. Barcelona: Paidós.

Rodrigo, M. y Palacios, J. (1998). Familia y desarrollo humano. Madrid: Alianza.

Romero, F. (2002). La mediación familiar. Un ejemplo de aplicación práctica: la comunicación a los hijos de la separación de los padres. El papel del mediador. Revista del Ministerio de Trabajo y Asuntos Sociales, 40, 31-54.

Sacristán, M. L. (1999). El lugar de Encuentro, un recurso en la gestión de los conflictos familiares. Ponencia I Congreso de Mediación Familiar. Valencia.

Sepúlveda, A. y Serrano, F. (2000). Punto de Encuentro familiar: Un recurso social alternativo. Apuntes de Psicología. 18 (2y3), 375-382. 